\title{
Numerical size comparisons in a phonologically transparent script
}

\author{
JYOTSNA VAID \\ University of California, San Diego, La Jolla, California \\ and Laboratory for Language and Cognitive Studies, The Salk Institute for Biological Studies, La Jolla, California
}

\begin{abstract}
When native readers of English are to judge which of two numbers is larger in value, their responses are influenced by the numbers' physical size. Interference occurs for digit and word modes of presentation when numerical and physical size cues conflict. The present study examined numerical size comparisons for digits and for words written in a syllabic script (Hindi) and in an alphabetic script (English). Significant interference was observed for digits and for English words only. The lack of interference for Hindi number words is discussed in terms of a greater reliance on phonological recoding in phonologically transparent scripts.
\end{abstract}

Cognitive psychologists have long been interested in how variations in the format of visual stimulation affect strategies of information processing. The question has been viewed from a number of different perspectives, including comparisons of word and picture (Banks \& Flora, 1977) and word and numeral processing (e.g., Hecaen \& Kremin, 1976). The primary concern of the present study was to determine whether different writing systems permit or encourage particular encoding strategies in accessing word meaning.

Writing systems are usually classified linguistically into three major categories, according to the size of the unit of writing. Logographic (or ideographic) scripts, such as Chinese, map onto speech at the level of the morpheme. Alphabetic writing systems map onto speech either at the phonemic level, as in Serbo-Croatian, or at the morphophonemic level, as in English. Syllabaries, which range from the Japanese kana to the Indian Devanagari scripts such as Hindi, map onto speech at the level of the syllable.

The purely formal characterization of the design of a script does not tell us whether there are differences across writing systems in, say, the way meaning is arrived at. The latter is essentially an empirical question. One way in which this question has been addressed has been to consider how the same information is coded by users of two different scripts. Perhaps the most extensively studied language in this regard has been Japanese, which contains a mixture of two scripts, one syllabic (kana) and the other ideographic (kanji). However, as Paradis, Hagiwara, and

A preliminary version of this paper, entitled "Skilled Processing of Number in a Semi-alphabetic Script," was presented at the annual meeting of the Psychonomic Society, San Diego, November 1983. The research described in this paper was conducted while the author was a National Institute of Mental Health postdoctoral trainee at Michigan State University.

Reprint requests may be directed to the author, c/o Department of Psychology, University of California, San Diego, C-009, La Jolla, CA 92093.
Hildebrandt (1985) have noted in a comprehensive review of the Japanese literature, there are various difficulties that arise in comparing performance with one script and that with another. Kanji, in isolation, are used to represent nouns, whereas kana characters are used to represent grammatical morphemes. Although everything in kanji could be written in kana (though it is usually not), the reverse is not possible: not everything can be written in kanji.

To properly study the influence of orthographic variation on lexical access, one must select a category of information that is equally likely to be found in one script as in another. The category of number meets this criterion, inasmuch as numbers can be written either as digits or as words, and number words are presumably used to the same extent in different writing systems.

Adapting a Stroop-like symbolic size-judgment paradigm first used to compare pictures and words (see Paivio, 1975), Besner and Coltheart (1979) sought to compare speeded judgments of numerical size of numbers presented in the form of digits or in the form of words. Subjects were shown pairs of numbers and were to decide which of the two numbers was larger in value. The numerically larger number was presented physically larger on half the trials and physically smaller on the remaining trials. Subjects were directed to ignore these variations in physical size. Besner and Coltheart found that mismatches in numerical and physical sizes resulted in slower response latencies only in the digit mode of number presentation; when the numbers were spelled out, variations in their physical size did not facilitate or inhibit judgments of numerical size.

On the basis of these results, Besner and Coltheart argued that mental comparison of numerical size for numbers presented in digit form proceeds directly from a visual representation of the input, and is thereby more susceptible to conflicting physical size cues. However, when numbers are presented in an alphabetic script, subjects are less susceptible to the interfering effects of phys- 
ical size presumably because the numbers are phonologically recoded before the comparison stage is reached.

Besner and Coltheart's finding of a differential Stroop effect for numbers presented as digits as opposed to words was recently called into question by Foltz, Poltrock, and Potts (1984), who pointed out that the stimulus set used in the spelled-out-numbers condition was smaller than that used in the digit condition, leading perhaps to a rapid learning of the correct response without the need to carry out a comparative judgment. In replicating the study, Foltz et al. matched the digit and word pairs on stimulus set size and found significant Stroop interference across both conditions. The size of the interference, although not directly compared, was still greater for digits than for words (Foltz et al., 1984, Table 3).

Although Besner and Coltheart's (1979) results may be suspect, their argument regarding the use of a visual versus a phonological representation of the input has been invoked in some recent cross-linguistic extensions of the number size paradigm in which stimulus set size was controlled. For example, Takahashi and Green (1983) obtained a Stroop effect that was larger for number pairs written in an ideographic script (kanji) than for numbers written in a syllabic script (kana). A similar effect was noted by Hatta (1983), who, in addition, studied visualfield asymmetries on this task. Vaid and Corina (1985) also examined visual-field differences in performance on numerical size judgments, comparing deaf and hearing users of American Sign Language. They found greater Stroop interference for left-visual-field presentation of digits. Tzeng and Wang (1983) reported a larger Stroop effect for Arabic numerals and for Chinese characters than for English number words.

Taken together, these studies concur in demonstrating that decisions about numerical size are more susceptible to variations in the physical parameters of the numbers when number words are presented in an ideographic script than when they are presented in alphabetic or syllabic scripts. This finding may, in tum, suggest that ideographic scripts encourage the use of direct visual coding even when, as in the number Stroop task, such a mode of representation is detrimental to performance.

Although studies of script-related differences in visual coding have for the most part involved comparisons of ideographic and alphabetic scripts (see Hung \& Tzeng, 1981 , for a review), there is some evidence to indicate that differences may be present even between alphabetic scripts. For example, Turvey, Feldman, and Lukatela (1984) present evidence to suggest that phonological recoding is a more common strategy for accessing words in Serbo-Croatian, an alphabetic script that is highly phonetic, than it is for processing words in English, an alphabetic script in which the grapheme-to-phoneme correspondence is often irregular. Differences in reading performance have also been noted between an alphabetic script and a syllabic script in some bilingual dyslexics (Karanth, 1981, 1982).
In view of the above, one may well expect differences in performance on the number Stroop task between alphabetic or syllabic scripts differing in phonological transparency in the direction of greater interference in the less phonetic script. Indeed, there is some evidence in support of this hypothesis, provided by a number Stroop experiment by Tzeng and Wang (1983) in which the languages compared were English and Spanish; a tendency was found for greater Stroop interference for English than for Spanish number words (Tzeng, personal communication, October 1983).

The present study sought to compare the performance of bilinguals in an alphabetic script (English) and in a syllabic script (Hindi). Hindi, which is written in the Devanagari script, resembles Serbo-Croatian in that it is so highly phonetic that it is rare to find exception words in the language (see Vaid, 1983). If presentation of number words in a phonologically transparent script, such as Hindi, encourages greater reliance on phonological recoding than is the case for a script such as English, in which the sound-to-symbol correspondence is less consistent, then one would expect the interference effect for numerical size comparisons to be smaller in Hindi than in English.

\section{METHOD}

\section{Subjects.}

The subjects were 10 college students from India studying at Michigan State University. They ranged in age from 23 to 35 years, with a mean of 27.6 years. The subjects' knowledge of written Hindi and written English was fairly advanced, since they had studied the two languages from early childhood and had used them extensively in and out of school. Hindi was the first language for half of the subjects; however, the other Indian languages known to the subjects were similar to Hindi in that they are written in Devanagari.

\section{Stimuli.}

Stimuli were selected from the numbers between 1 through 9 that are monosyllabic in English (thus, the number 7 was excluded). The following pairs of numbers were used: 5 and 8,1 and 6,2 and 3 , and 4 and 9 . Each stimulus pair was presented in three forms-as digits, as words in English, and as words in Hindi. Number words in English were written in capital letters; there is no comparable distinction in Hindi. For both languages, stimulus pairs were displayed horizontally, the numbers in each pair being separated by about $1 \mathrm{~cm}$. Physical size of the numbers varied such that the numerically larger number was written physically larger on half of the trials, and physically smaller on the remaining half. For all three modes of presentation, the physically larger stimuli were about twice the size of the physically smaller stimuli (see Figure 1).

\section{Procedure}

Congruent and incongruent versions of each of the stimulus pairs were presented twice, once with the physically larger number to the left of the other number, and once with it to the right of the other number, making for a total of 16 trials per mode of presentation. All subjects were tested on each mode, with half receiving the digit condition before the word condition, and the remainder receiving the reverse. In the word condition, the English and Hindi words were randomized.

Slides of the stimuli were presented in a projection tachistoscope for an exposure duration of $300 \mathrm{msec}$. The subjects' task was to 


\section{INCONGRUENT}

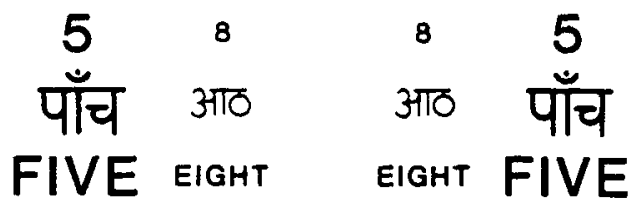

\section{CONGRUENT}

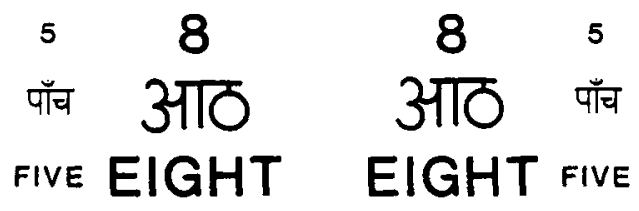

Figure 1. Sample stimulus pairs used in experiment.

make a speeded decision as to which of the two numbers on any given trial was numerically the larger. Stimulus onset triggered a digital timer, accurate to the millisecond, which was deactivated by the subject's vocal response.

\section{RESULTS}

Reaction-time scores for correct responses were entered into a two-way analysis of variance as a function of congruency (that is, a match or a mismatch in physical and numerical size) and mode of presentation (Arabic numerals, English words, Hindi words). Mean response latencies as a function of congruency and presentation mode are summarized in Table 1 . The data analysis revealed main effects for congruency $[F(1,9)=6.86$, $p<$ $.03]$ and presentation mode $[F(2,18)=32.91$, p < $.00001]$ and a significant interaction of presentation mode $x$ congruency $[\mathrm{F}(2,18)=8.56, \mathrm{p}<.002]$. Interaction breakdown indicated that the congruency effect was significant for digits and English words only; Hindi words showed no significant differences in latency between incongruent and congruent stimuli.

Table 1

Mean Performance (in Milliseconds) and Standard Deviations for Numerical Size Comparisons as a Function of Congruency and Presentation Mode

\begin{tabular}{lccccc} 
& \multicolumn{2}{c}{ Incongruent } & & \multicolumn{2}{c}{ Congruent } \\
\cline { 2 - 3 } \cline { 5 - 6 } & Mean & SD & & Mean & SD \\
\hline Arabic Numerals & 642 & 109 & $601^{*}$ & 81 \\
English Words & 787 & 87 & $731^{*}$ & 102 \\
Hindi Words & 801 & 127 & 818 & 138 \\
\hline
\end{tabular}

$\overrightarrow{\text { Note }-N}=10 . \quad$ *The difference in response latency between incongruent and congruent stimuli was significant at or beyond the .05 level.

\section{DISCUSSION}

The findings from this study are of relevance to the question of whether writing systems differ in terms of the kinds of encoding strategies they permit or encourage in accessing meaning. While decisions about numerical size were fastest in the Arabic numeral condition, physical size cues influenced judgments of numerical size to about the same extent in the numeral and English word conditions. This would suggest that a visual representation of the input was used in performance on the task. In contrast, Hindi number size judgments were not influenced by the words' physical size. (Inspection of individual subjects' data revealed that there were no differences between native and nonnative Hindi users in this regard.) This finding is as predicted and suggests that phonological encoding was a consistent strategy used by subjects when making numerical size comparisons in Hindi.

The finding of a reduced Stroop interference effect in Hindi relative to English suggests that, when script-tosound mapping is direct and regular, phonological recoding may be the preferred route for lexical access. In contrast, use of such a strategy for processing written English does not appear to be the preferred route on this task (or, perhaps, on other tasks as well; see McCusker, Hillinger, \& Bias, 1981). Whether phonological recoding of English is used on the number Stroop task or not appears to depend on the characteristics of the contrasting orthography used in the experiment, ideographic (as in Tzeng \& Wang, 1983) or syllabic (as in the present experiment).

A general implication of the present study is that, in addition to comparisons between ideographic and alphabetic scripts, differences in processing between alphabetic and syllabic scripts need also to be considered.

\section{REFERENCES}

BANKs, W., \& FLORA, J. (1977). Semantic and perceptual processes in symbolic comparisons. Journal of Experimental Psychology: Human Perception and Performance, 3, 278-290.

Besner, D., \& Coltheart, M. (1979). Ideographic and alphabetic processing in skilled reading of English. Neuropsychologia, 17, 467-472.

Foltz, G., Poltrock, S., \& Potrs, G. (1984) Mental comparison of size and magnitude: Size congruity effects. Journal of Experimental Psychology: Learning, Memory and Cognition, 10, 442-453.

HATTA, T. (1983). Visual field differences in semantic comparative judgments with digits and kanji stimulus materials. Neuropsychologia, 21, 669-678.

HeCAEN, H., \& KREMIN, H. (1976). Neurolinguistic research on reading disorders resulting from left hemisphere lesions. In $\mathbf{H}$. Whitaker \& H. A. Whitaker (Eds.), Studies in neurolinguistics (Vol. 2). New York: Academic Press.

HuNG, D., \& TzENG, O.(1981). Orthographic variations and visual information processing. Psychological Bulletin, 90, 377-414.

Karanth, P. (1981). Pure alexia in a Kannada-English bilingual. Cortex, 17, 187-198.

Karanth, P. (1982). Dyslexia in a Dravidian language. Paper presented at a seminar on "Surface Dyslexia" at Oxford University.

McCusker, L., Hilingaer, M., \& Bias, R. (I981). Phonological recoding and reading. Psychological Bulletin, 89, 214-245.

Paivio, A. (1975). Perceptual comparisons through the mind's eye. Memory \& Cognition, 3, 635-647. 
Paradis, M., Hagiwara, H., \& Hildebrandt, N. (I985). Neurolinguistic aspects of the Japanese writing system. Orlando, FL: Academic Press.

TAKahashi, A., GREEN, D. (I983). Numerical judgments with kanji and kana. Neuropsychologia, 21, 259-263.

Turvey, M., Feldman, L., \& Lukatela, G. (1984). The SerboCroation orthography constrains the reader to a phonologically analytic strategy. In L. Henderson (Ed.), Orthographies and reading: Perspectives from cognitive psychology, neuropsychology, and linguistics (pp. 81-90). London: Erlbaum.

Tzeng, O., \& WANG, W. S.-Y. (I983). The first two R's. American Scientist, 71, 238-243.
VAID, J. (1983). Reading processes in Hindi: A preliminary investigation. Paper presented at annual meeting of the South Asian Languages Analysis Roundtable, University of Mlinois, Urbana.

VAID, J., \& CORINA, D. (1985). Visual field asymmetries in numerical size judgments for digits, words, and ASL. Paper presented at International Neuropsychological Society, Eighth European Conference, Copenhagen.

(Manuscript received December 3, 1984; revision accepted for publication May 30, 1985. 